\title{
ASYMPTOTICS OF THE DENSITY OF THE SUPREMUM OF A RANDOM WALK WITH HEAVY-TAILED INCREMENTS
}

\author{
YUEBAO WANG, * Soochow University \\ KAIYONG WANG, ${ }^{* *}$ University of Science and Technology of Suzhou
}

\begin{abstract}
Under some relaxed conditions, in this paper we obtain some equivalent conditions on the asymptotics of the density of the supremum of a random walk with heavy-tailed increments. To do this, we investigate the asymptotics of the first ascending ladder height of a random walk with heavy-tailed increments. The results obtained improve and extend the corresponding classical results.
\end{abstract}

Keywords: Random walk; heavy-tailed increment; first ascending ladder height; density of the supremum; asymptotics

2000 Mathematics Subject Classification: Primary 60G50; 60F99; 60E05

\section{Introduction}

It is well known that the asymptotics of the density of the supremum of a random walk with heavy-tailed increments is an important subject to which researchers pay close attention. Here we present some equivalent conditions on such asymptotics, under some relaxed conditions.

Let $\left\{\xi_{i}: i \geq 1\right\}$ be independent, identically distributed proper random variables with common distribution $F$ on $(-\infty, \infty)$ and finite negative mean $\mu_{F}=-m$. Write

$$
\bar{F}(x)=1-F(x), \quad x \in(-\infty, \infty) .
$$

Let $S_{0}=0$ and $S_{n}=\sum_{i=1}^{n} \xi_{i}, n \geq 1$, define a random walk. Then $M=\sup _{n \geq 0} S_{n}$, the supremum of the random walk, is almost surely a random variable, with distribution $W$ such that $0<p=\bar{W}(0)<1$. If $W$ is absolutely continuous, let $w$ denote its density.

Because the asymptotics of $W$ is related to the first ascending ladder height of the random walk, we give some concepts about, and notation for, the latter. Let

$$
\tau_{+}=\inf \left\{n \geq 1: S_{n}>0\right\}
$$

be the first ascending ladder epoch of the above random walk. It is well known that, when $m=-\mu_{F}>0, \tau_{+}$is a defective random variable, i.e. $\mathrm{P}\left(\tau_{+}<\infty\right)<1$. Let $\left\{\eta_{i}: i \geq 1\right\}$ be independent proper random variables with common distribution $G$ such that

$$
G(x)=\mathrm{P}\left(\eta_{1} \leq x\right)=\mathrm{P}\left(S_{\tau_{+}} \leq x \mid \tau_{+}<\infty\right)
$$

Received 19 September 2005; revision received 5 April 2006.

* Postal address: Department of Mathematics, Soochow University, Suzhou, 215006, P. R. China.

Email address: ybwang@suda.edu.cn

** Postal address: Department of Applied Mathematics, University of Science and Technology of Suzhou, Suzhou, 215009, P. R. China. 
here $S_{\tau_{+}}$is (said to be) the first ascending ladder height of the above random walk. If $G$ is absolutely continuous, let $g$ denote its density.

Since the asymptotics of $W$ is also related to $F$, we introduce some common distribution classes. To do so, we assume that, unless stated otherwise, all limiting relationships in this paper hold as $x \rightarrow \infty$. If $a(x) b(x)^{-1} \rightarrow 1$, we write $a(x) \sim b(x)$; if $\lim _{\sup _{x \rightarrow \infty}} a(x) b(x)^{-1}<\infty$, we write $a(x)=O(b(x))$; and if $a(x)=O(b(x))$ and $b(x)=O(a(x))$, we write $a(x) \approx b(x)$. A nonnegative function $l$ on $(-\infty, \infty)$ is said to be long tailed, written $l \in \mathcal{L} d$, if $l$ is eventually positive and $l(x+y) \sim l(x)$ uniformly for $|y| \leq 1$. If

$$
l \in \mathcal{L} d, \quad \int_{0}^{\infty} l(y) \mathrm{d} y<\infty, \quad \text { and } \quad \int_{0}^{x} l(x-y) l(y) \mathrm{d} y \sim 2 \int_{0}^{\infty} l(y) \mathrm{d} y l(x),
$$

then we write $l \in \delta d$. For a proper distribution $L$ on $(-\infty, \infty)$, if $1-L=\bar{L} \in \mathcal{L} d$ then we write $L \in \mathcal{L}$; if $\bar{L} \in \delta d$ then we write $L \in \mathcal{s}^{*}$; and if $\int_{0}^{x} \bar{L}(x-y) L(\mathrm{~d} y) \sim 2 \bar{L}(x)$ then we write $L \in \delta$. It is well known that $\delta$ and $\delta^{*}$ are the spaces introduced by Chistyakov (1964) and Klüppelberg (1988), respectively. They are two of the most important heavy-tailed distribution classes and have the following proper relationship: $8^{*} \subset \delta \subset \mathcal{L}$. Finally, for a proper distribution $L$ on $(-\infty, \infty)$, let

$$
\overline{L^{I}}(x)=\min \left\{1, \int_{x}^{\infty} \bar{L}(y) \mathrm{d} y\right\}, \quad x \geq 0 .
$$

Theorem 1.1. Assume that $F \in \mathcal{L}$ has density $f(x)=f_{1}(x) f_{2}(x), x \in(-\infty, \infty)$, where $f_{1} \in \mathcal{L} d$ and $f_{2}$ is an eventually positive, nonincreasing function. Then $G$ and $W$ are absolutely continuous and the following statements are equivalent: (i) $F \in \varsigma^{*}$; (ii) $g \in \delta d$; (iii) $w \in \delta d$; (iv) $w(x) \sim m^{-1} \bar{F}(x)$.

Remark 1.1. Under the conditions that $F \in \mathcal{S}^{*}$ and $f \in \mathcal{L} d$, Theorem 4 of Asmussen et al. (2003) stated part (iv) of our Theorem 1.1. Obviously, the latter theorem improves and extends this result. However, we must point out that the idea of the proof comes from Asmussen et al. (2003) and Asmussen et al. (2002). The main difference is that we divide $f$ into two factors.

In Section 2 we will prove Theorem 1.1. For this, we need a lemma on the asymptotics of the first ascending ladder height. In Section 3 we will extend the lemma of Section 2 and show that it is independently significant and can be used to give new proofs of some classical results.

\section{Proof of Theorem 1.1}

We use the following lemma to prove Theorem 1.1.

Lemma 2.1. Under the conditions of Theorem 1.1 we have

$$
\begin{aligned}
I(x) & :=\sum_{n=1}^{\infty} \mathrm{E} f\left(x-S_{n}\right) \mathbf{1}\left(A_{n}\right) \\
& \sim(1-p) m^{-1} \bar{F}(x),
\end{aligned}
$$

where $A_{n}=\left\{S_{j} \leq 0: j=0, \ldots, n\right\}, n \geq 0$.

Proof. We consider the taboo renewal function $H(B)=\sum_{n=0}^{\infty} H_{n}(B), B \subset(-\infty, 0]$, where $H_{0}(B)=\mathbf{1}(0 \in B)$ and $H_{n}(B)=\mathrm{P}\left(A_{n}, S_{n} \in B\right), n \geq 1$. According to Blackwell's 
renewal theorem, for each $a \in(-\infty, \infty)$ we have

$$
H(-x+a)-H(-x) \rightarrow m^{-1} a(1-p) .
$$

For sufficiently large $x$, since $f_{1} \in \mathcal{L} d$ and $f_{2}$ is eventually nonincreasing, we have

$$
\begin{aligned}
I(x) & =-\sum_{j=0}^{\infty} \int_{j}^{j+1} f_{1}(x+z) f_{2}(x+z) H(-\mathrm{d} z) \\
& \sim-\sum_{j=0}^{\infty} f_{1}(x+j) \int_{j}^{j+1} f_{2}(x+z) H(-\mathrm{d} z) \\
& \leq \sum_{j=0}^{\infty} f(x+j)(H(-j)-H(-j-1))
\end{aligned}
$$

and

$$
\begin{aligned}
I(x) & \sim-\sum_{j=0}^{\infty} f_{1}(x+1+j) \int_{j}^{j+1} f_{2}(x+z) H(-\mathrm{d} z) \\
& \geq \sum_{j=0}^{\infty} f(x+1+j)(H(-j)-H(-j-1)) .
\end{aligned}
$$

Since $f_{1} \in \mathcal{L} d$ we furthermore know that

$$
\begin{aligned}
\mathscr{H}\left(f_{1}\right): & =\left\{h(x):[0, \infty) \rightarrow[0, \infty): h(x) \uparrow \infty, x^{-1} h(x) \rightarrow 0,\right. \text { and } \\
& \left.f_{1}(x-y) \sim f_{1}(x) \text { uniformly for }|y| \leq h(x)\right\} \\
& \neq \varnothing,
\end{aligned}
$$

and if $h \in \mathcal{H}\left(f_{1}\right)$ then, for any $h_{1}:[0, \infty) \rightarrow[0, \infty)$, if $h_{1}(x) \uparrow \infty$ and $h_{1}(x) \leq h(x)$, we have $h_{1} \in \mathcal{H}\left(f_{1}\right)$ (see Foss and Zachary (2003), for example). We thus can choose an integer-valued function $h \in \mathscr{H}\left(f_{1}\right)$ such that

$$
f(x) h(x)=o(\bar{F}(x)) \text { and } \quad h(x)=O(h(x-1)) .
$$

By (2.2), Toeplitz's lemma (see Stout (1974, p. 120)), and (2.5), when $x$ is sufficiently large we have

$$
\begin{aligned}
\sum_{j=0}^{h(x)} f(x+j)(H(-j)-H(-j-1)) & \sim f_{1}(x) \sum_{j=0}^{h(x)} f_{2}(x+j)(H(-j)-H(-j-1)) \\
& \leq f(x) h(x) h(x)^{-1} \sum_{j=0}^{h(x)}(H(-j)-H(-j-1)) \\
& \sim(1-p) m^{-1} f(x) h(x) \\
& =o(\bar{F}(x)) .
\end{aligned}
$$


Furthermore, by (2.2), (2.5), and $F \in \mathcal{L}$, when $x$ is sufficiently large we have

$$
\begin{aligned}
& \sum_{j=h(x)+1}^{\infty} f(x+j)(H(-j)-H(-j-1)) \\
& \sim(1-p) m^{-1} \sum_{j=h(x)+1}^{\infty} f_{1}(x+j) f_{2}(x+j) \\
& \leq(1-p) m^{-1} \sum_{j=h(x)+1}^{\infty} f_{1}(x+j) \int_{j}^{j+1} f_{2}(x-1+y) \mathrm{d} y \\
& \sim(1-p) m^{-1} \sum_{j=h(x)+1}^{\infty} \int_{j}^{j+1} f(x-1+y) \mathrm{d} y \\
& \leq(1-p) m^{-1} \int_{0}^{\infty} f(x-1+y) \mathrm{d} y .
\end{aligned}
$$

It follows from (2.3), (2.6), (2.7), and $F \in \mathcal{L}$ that

$$
\limsup _{x \rightarrow \infty} I(x) \bar{F}(x)^{-1} \leq(1-p) m^{-1} .
$$

Similarly, using (2.4) we can prove that

$$
\liminf _{x \rightarrow \infty} I(x) \bar{F}(x)^{-1} \geq(1-p) m^{-1} .
$$

Thus, (2.1) follows from (2.8) and (2.9).

Proof of Theorem 1.1. By definition of $\tau_{+}$, we have

$$
\begin{aligned}
\bar{G}(x) & =\mathrm{P}\left(S_{\tau_{+}}>x \mid \tau_{+}<\infty\right) \\
& =p^{-1} \sum_{n=1}^{\infty} \mathrm{P}\left(S_{n}>x, A_{n-1}\right) \\
& =p^{-1} \sum_{n=1}^{\infty} \int_{-\infty}^{0} \int_{x-y}^{\infty} f(z) \mathrm{d} z \mathrm{dP}\left(S_{n-1} \leq y, A_{n-1}\right) .
\end{aligned}
$$

Thus, $G$ is absolutely continuous and, by Lemma 2.1 , we know that its density is

$$
\begin{aligned}
g(x) & =p^{-1} \sum_{n=1}^{\infty} \int_{-\infty}^{0} f(x-y) \mathrm{dP}\left(S_{n-1} \leq y, A_{n-1}\right) \\
& =p^{-1} \int_{-\infty}^{0} f(x-y) H(\mathrm{~d} y) \\
& =p^{-1} I(x) \\
& \sim(1-p) m^{-1} p^{-1} \bar{F}(x) .
\end{aligned}
$$

It follows from (2.10) that (i) $\Longleftrightarrow$ (ii). Also by (2.10), we know that $g \in \mathcal{L} d$. Thus, by Theorem 3.2 of Klüppelberg (1989), we have (ii) $\Longleftrightarrow$ (iii) $\Longleftrightarrow$ (iv), completing the proof. 


\section{Extension of Lemma 2.1 and applications}

Lemma 2.1 is the key to proving Theorem 1.1. In fact, Lemma 2.1 is of independent significance. In order to explain this, we extend Lemma 2.1 and obtain a general result. For any given function $u$ and some $T, 0<T \leq \infty$, let $v(x):=u(x)-u(x+T), U(x):=\int_{x}^{\infty} u(y) \mathrm{d} y$, and $V(x):=U(x)-U(x+T)$, for $x \in(-\infty, \infty)$. We then have the following proposition.

Proposition 3.1. Assume that $U(0)<\infty$ and $V \in \mathcal{L} d$; that $u$ on $(-\infty, \infty)$ is an eventually positive, nonincreasing function for $0<T<\infty$; and that $u(x)=u_{1}(x) u_{2}(x), x \in(-\infty, \infty)$, for $T=\infty$, where $u_{1} \in \mathcal{L} d$ and $u_{2}$ is an eventually positive, nonincreasing function. Then

$$
I(x):=\sum_{n=1}^{\infty} \mathrm{E} v\left(x-S_{n}\right) \mathbf{1}\left(A_{n}\right) \sim(1-p) m^{-1} V(x),
$$

where $A_{n}=\left\{S_{j} \leq 0: j=0, \ldots, n\right\}, n \geq 0$.

If we take $v=f$ and $T=\infty$ in Proposition 3.1, then we recover Lemma 2.1. If we take $u=\bar{F}$ in Proposition 3.1, then we obtain the following result.

Corollary 3.1. Let $V(x)=\overline{F^{I}}(x)-\overline{F^{I}}(x+T), x \in(-\infty, \infty)$, for some $T, 0<T \leq \infty$. Assume that $V \in \mathcal{L} d$. Then

$$
\left.\bar{G}(x)-\bar{G}(x+T) \sim(1-p) p^{-1} m^{-1} \overline{\left(F^{I}\right.}(x)-\overline{F^{I}}(x+T)\right) .
$$

In order to prove Proposition 3.1 we need the following lemmas. We omit their proofs.

Lemma 3.1. Assume that $u(x)=u_{1}(x) u_{2}(x), x \in(-\infty, \infty)$, where $u_{1} \in \mathcal{L} d$ and $u_{2}$ is an eventually positive, nonincreasing function. For $0<T<\infty$, we have $u \in \mathcal{L} d \Longleftrightarrow V \in \mathcal{L} d$, each side of which implies that $V(x) \sim T u(x)$. For $T=\infty$, we have $U \in \mathcal{L} d \Longleftrightarrow u(x)=$ $o(U(x))$.

Lemma 3.2. Assume that $U(0)<\infty$ and that $u$ satisfies the condition of Lemma 3.1. Then $u(x) \rightarrow u(\infty)=v(\infty)=U(\infty)=V(\infty)=0$ and $V(x)=U(x)$, for $T=\infty$.

Proof of Proposition 3.1. For $T=\infty$, the proof is similar to the proof of Lemma 2.1. For $0<T<\infty$, we can prove the proposition using Lemma 3.1 and Lemma 3.2, following the lines of the proof of Lemma 3 of Asmussen et al. (2002). We omit the details.

In Corollary 3.1, from (3.1) and Lemma 3.1 we have

$$
\bar{G}(x)-\bar{G}(x+T) \sim T(1-p) p^{-1} m^{-1} \bar{F}(x)
$$

for $0<T<\infty$; (3.2) is Lemma 3 of Asmussen et al. (2002). Furthermore, if $F \in 8^{*}$ then, using a standard method, it is easy to prove that

$$
\bar{W}(x)-\bar{W}(x+T) \sim m^{-1} T \bar{F}(x),
$$

which is Theorem 1 of Asmussen et al. (2002).

In Corollary 3.1, from Lemma 3.2, if $U=V=F^{I} \in \mathcal{L}$ then

$$
\bar{G}(x) \sim(1-p) p^{-1} m^{-1} \overline{F^{I}}(x)
$$


for $T=\infty$. Thus, we obtain another known result, Theorem 10 of Borovkov (1976, Chapter 4), by a different method. Furthermore, if $F^{I} \in \delta$ then, using (3.3) and a standard method, it is easy to prove that

$$
\bar{W}(x) \sim m^{-1} \bar{F}(x) .
$$

It is well known that (3.4) is Theorem 2(B) of Veraverbeke (1977).

We expect that for different choices of $u$ we can obtain other results.

\section{Acknowledgements}

The authors would like to express their deep gratitude to the referee for constructive comments on the previous versions of this paper. This work was supported by the National Science Foundation of China (grant number 10271087).

\section{References}

Asmussen, S., Foss, S. And Korshunov, D. (2003). Asymptotics for sums of random variables with local subexponential behavior. J. Theoret. Prob. 16, 489-518.

Asmussen, S. et al. (2002). A local limit theorem for random walk maxima with heavy tails. Statist. Prob. Lett. 56, 399-404.

Borovkov, A. A. (1976). Stochastic Processes in Queueing. Springer, New York.

Chistyakov, V. P. (1964). A theorem on the sums of independent positive random variables and its applications to branching random processes. Theory Prob. Appl. 9, 640-648.

Foss, S. AND Zachary, S. (2003). The maximum on a random time interval of a random walk with long-tailed increments and negative drift. Ann. Appl. Prob. 13, 37-53.

KlüPPELberg, C. (1988). Subexponential distributions and integrated tails. J. Appl. Prob. 25, 132-141.

KlÜPPELberg, C. (1989). Subexponential distributions and characterization of related classes. Prob. Theory Relat. Fields 82, 259-269.

Stout, W. F. (1974). Almost Sure Convergence. Academic Press, New York.

Veraverbeke, N. (1977). Asymptotic behavior of Wiener-Hopf factors of a random walk. Stoch. Process. Appl. 5, 27-37. 